\title{
Maximum Load Balancing with Optimized Link Metrics
}

\author{
Touraj Shabanian, Massoud Reza Hashemi, Ahmad Askarian
}

Department of Electrical and Computer Engineering, Isfahan University of Technology, Isfahan, Iran.

Email: shabanian@ec.iut.ac.ir, hashemim@cc.iut.ac.ir, a.askarian@ec.iut.ac.ir

Received 2012

\begin{abstract}
Traffic engineering helps to use network resources more efficiently. Network operators use TE to obtain different objectives such as load balancing, congestion avoidance and average delay reduction. Plane IP routing protocols such as OSPF, a popular intradomain routing protocol, are believed to be insufficient for TE. OSPF is based on the shortest path algorithm in which link weights are usually static value without considering network load. They can be set using the inverse proportional bandwidth capacity or certain value. However, Optimization theory helps network researchers and operators to analyze the network behavior more precisely. It is not a practical approach can be implemented in traditional protocol .This paper proposes that to address the feasibility requirements, a weight set can be extracted from optimization problem use as a link metric in OSPF. We show the routes that selected in OSPF with these metric distribute the traffic more close to optimal situation than routes from OSPF with default metric.
\end{abstract}

Keywords: Component; Formatting; Style; Styling; Insert

\section{Introduction}

This In recent years, the use of the Internet as communication infrastructure for different telecommunication applications has been growing significantly. Because bandwidth is one of the most important requirements of these applications, network hardware should support bandwidth management techniques. Traffic engineering (TE) is a bandwidth management technique that considers different objectives such as maximum throughput, minimum congestion and load balancing in the network. TE puts the traffic where network bandwidth is available [1]. TE with the objective of load balancing can reduce maximum link utilization (MLU) and increase bandwidth efficiency (BWE). Because considerable delay may occur at congested links, reduction of end to end delay can be achieved as a side result of load balancing.

Destination-based routing is not flexible for TE, and so it is highly susceptible to congestion. Because of this reason the concept of TE was developed mostly in MPLS-based networks [2,3]. MPLS-based TE can optimize traffic distribution using dedicated label switch paths (LSP). The capability of explicit routing and arbitrary traffic splitting are the most important features of MPLS TE. But the MPLS has not been widely deploy Rapid increase in network traffic especially that of new applications which require QoS guarantees, has encouraged the network providers to apply IP based TE with different objectives. The main idea of IP-based TE is to find a set of weights that optimizes a specific objective function. If the objective function is the total link cost, the constraint of equal cost multipath (ECMP) causes the problem to be NP hard [4]. Different near-optimal heuristic algorithms based on local search were proposed to solve this problem [4].

One approach for analyzing the TE problem is formulating it with optimization theory problems. If we consider load-balancing as an objective of the optimization problem and consider the amount of traffic load on all links that belong to a specific session as the problem outcome, the solution of such problem is the path of each session that results in minimum congestion.

Measurements in [5] indicate that bottlenecks of the Internet backbone are not only located between ASs but also they exist in intradomain links. The popular intradomain routing protocol is OSPF. In this paper we present a formulation of the optimization problem that object to provide maximum load balancing. This objective function is useful in a situation that network entrance is random since increase the probability of new traffic admission. In addition we try to extract the OSPF metric from this problem and therefore reach the load balancing with OSPF routing. These attempts result in a new definition such as equivalent weight set and equivalent constraints. In this paper we analyze the optimization problem from feasibility perspective and show that a set of link weights that can be embedded as a link metric in OSPF protocol results in optimal or near optimal load balancing. Our simulations show that this method im- 
proves bandwidth efficiency and reduces network congestion and also leads to a substantial reduction in the end to end delay.

\section{Problem Statement}

First, Different TE objectives lead to different objective functions of optimization problem. We consider load balancing as an objective of traffic engineering so the objective function of the optimization problem is to minimize MLU (maximum link utilization). Consider the linear optimization problem that is called first primal problem (PRIMAL_I) with the following notation. A connected graph $G(N, A)$ is given. $c_{i j}:(i, j) \in A$ is a set of edge capacities and $\left(s_{k}, d_{k}\right)$ is a set of sourcedestination pairs for each session $k \in K$. $D_{k}$ Is the total amount of session ${ }^{k}$ traffic. The amount of traffic in link $(i, j) \in A$ that belongs to session $k$ is $X_{i j}^{k}$. So the problem is:

$$
\begin{aligned}
& \min M L U \\
& \sum_{j:(i, j) \in A} X_{i, j}^{k}-\sum_{j:(j, i) \in A} X_{j, i}^{k}= \begin{cases}D_{i} & i=\text { sourse } \\
-D_{i} & i=\text { destination } \\
0 & \text { o.w }\end{cases} \\
& \sum_{k \in K} X_{i, j}^{k} \leq C_{i, j} \cdot M L U \\
& X_{i, j} \geq 0
\end{aligned}
$$

Constraints (2) are flow conservation constraints that are derived from network topology. Constraint (3) ensure that link flows do not violate link capacity and (4) says that link flows are nonnegative. The PRIMAL_I solution specifies the $X_{i j}^{k}$, and so we have the optimal path with arbitrary splitting for all sessions that minimize MLU. Our objective is to find a practical method suited for IP networks that forces the traffic to go through a set of optimal paths. To achieve this goal we should find a set of new link metrics such that all paths which are specified by PRIMALI problem can also be obtained by the shortest path algorithm in regard to the new metrics. It means that if $X_{i j}^{k}>0$, then link $(i, j)$ should be selected by session $\mathrm{k}$ according to the shortest path algorithm. Here we assume that the shortest path algorithm is OSPF that supports Equal-Cost Multi-Path (ECMP).

The load balancing methods introduced in [6,7] are based on primal optimization problem. In this paper we consider the dual optimization problem (DUAL_II) which is obtained with respect to Lagrange Multipliers. In other word we aim to distribute traffic by determining the links weight. And It will be shown that the Lagrange Multipliers comparable with constraint (3) can be interpreted as OSPF link metrics that satisfy the load balancing objective. Link metric in OSPF protocol must be an integer between 1 and 65535 but we will show in section IV that the Lagrange Multipliers that are obtains from the solution of DUAL_II problem and comparable with constraint (3), do not satisfy this range in general. So the following definition gives us the choice of an alternative weight set.

Definition 1: Two weights set $\vec{W}=\left\{w_{i}\right\}_{i=1}^{L}$ and $\vec{W}^{\prime}=\left\{w_{i}^{\prime}\right\}_{i=1}^{L}$ are equivalent with respect to a given graph $G(N, A)$ with $L$ links, if and only if the shortest paths between any arbitrary nodes in $G$ are the same considering any one of these two weight sets.

\section{The Dual Problem}

Before Before Defining the Lagrange multiplayer $\left\{p_{i}\right\}_{i=1}^{N}$ comparable with constraint (2) and $\left\{w_{i, j}\right\}_{(i, j) \in A}$ comparable with constraint (3), the Lagrange polynomial is:

$$
\begin{aligned}
& \underset{X_{i j \geq 0}^{k} \geq 0}{L}(X, M L U, P, W) \\
= & M L U+\sum_{k \in K} \sum_{i \in N} p_{i}\left(D_{k}-\sum_{j:(i, j) \in A} X_{i j}^{k}+\sum_{j:(j, i) \in A} X_{j i}^{k}\right) \\
& +\sum_{(i, j) \in A} w_{i j}\left(\sum_{k \in K} X_{i j}^{k}-C_{i j}\right)
\end{aligned}
$$

For more details can be referred to chapter 5 of [9].To achieve the dual problem the following equation should be satisfied for each feasible $X$ and $M L U$.

$$
M L U \geq L(X, M L U, P, W)
$$

To satisfy (6) we must have: $w_{i j} \geq 0$. Now the function $g(W, P)$ is defined as bellow:

$$
g(W, P)=\min _{X, M L U} L(X, M L U, P, W)
$$

So we have:

$$
\begin{aligned}
g(W, P)= & \min _{X, M L U} \sum \sum p_{i}^{k} D_{i}^{k} \\
& +\sum_{k \in K} \sum_{(i, j) \in A} X_{i j}^{k}\left(p_{j}-p_{i}+w_{i j}\right) \\
& +M L U\left(1-\sum_{(i, j) \in A} w_{i j} C_{i j}\right)
\end{aligned}
$$

Because $w_{i j}$ and $X_{i j}^{k}$ are positive values, we have:

$$
g(W, P)=\left\{\begin{array}{lc}
\sum_{k \in K} \sum_{i \in N} p_{i}^{k} D_{i}^{k} & p_{i}-p_{j} \leq w_{i j} \text { and } \sum C_{i j} w_{i j}=1 \\
-\infty & p_{i}-p_{j} \geq w_{i j} \text { or } \sum_{(i, j) \in A} C_{i j} w_{i j} \neq 1
\end{array}\right.
$$

The dual function is defined to maximize $g(W, P)$ when all $w_{i j}$ are positive values. Equation (10) shows the DUAL_I problem that is the dual function of PRIMAL_I.

$$
\begin{gathered}
\max \sum_{k \in K} p_{t_{k}}^{k} \cdot D^{k} \\
p_{i}^{k}-p_{j}^{k} \leq w_{i j} \\
\sum_{(i, j) \in A} C_{i j} w_{i j}=1
\end{gathered}
$$




$$
\begin{aligned}
& p_{s_{k}}^{k}=0 \\
& w_{i j} \geq 0
\end{aligned}
$$

As the primal and dual problems are linear, strong duality holds and according to complementary slackness in KKT theorem if $\hat{X}_{i j}^{k}$ is optimal solution of PRIMAL_I and $\left\{\hat{w}_{i j}, p_{i j}^{k}\right\}$ is the optimal solution of DUAL_I we have:

$$
\hat{X}_{i j}^{k} \cdot\left(\hat{p}_{i}^{k^{\wedge}}-p_{j}^{k}+w_{i j}\right)=0
$$

Equation (15) indicates that if session $k$ passes link $(i, j)$ then $p_{j}^{k}-p_{i}^{k}=w_{i j}$. According to theorem 1 in [8] if $\left\{\hat{w}_{i j}\right\}_{(i, j) \in A}$ is used as a link metric in a shortest path algorithm, all non empty links $\left(X_{i j}^{k}>0\right)$ will be included among the selected paths by the shortest path algorithm procedure.

\section{Practical Requirements}

$\left\{w_{i j}\right\}_{(i, j) \in A}$ that is calculated from the DUAL_I are equal to or greater than zero. But as we mentioned before OSPF link metrics cannot be zero. We show that there exists a weight set equivalent to $\left\{w_{i j}\right\}_{(i, j) \in A}$ that can be obtained using the new optimization problem.

Proposition 1: consider $G(N, A)$ with weight set $\left\{w_{i j}\right\}_{(i, j) \in A}$ and some scalars $\left\{\delta_{i}\right\}_{i=1}^{N}$ corresponding to each link and node respectively. If we change the link weight to $\bar{w}_{i j}=w_{i j}+\delta_{j}-\delta_{i}$, then the weight set $\left\{w_{i j}\right\}_{(i, j) \in A}$ and $\left\{\bar{w}_{i j}\right\}_{(i, j) \in A}$ are equivalent weight sets with respect to $G(N, A)$ [8]. To achieve non-zero weight set we changed the weights of the links according to algorithm 1.

Algorithm 1:

Step 1: For each session $k \in K$ assign the scalar set $\left\{\delta_{i}^{k}\right\}_{i=1}^{N}$ as follows:

- If there exists at least one directed path to node i from source node of session $\mathrm{k}\left(s^{k}\right)$, then $\delta_{i}^{k}$ is equal to the length of the longest hop-count non-loopy path from $s^{k}$ to i.

- Else $\delta_{i}^{k}$ is equal to zero.

Step 2: Assign the $\max _{k} \delta_{i}^{k}$ as the final scalar $\delta_{i}$ Step 3:

- If the $\delta_{j}-\delta_{i} \geq 0$ then $\bar{w}_{i j}=w_{i j}+\delta_{j}-\delta_{i}$

- Else $\bar{w}_{i j}=w_{i j}+1$.

So according to Proposition 1 and algorithm 1, there exists an equivalent weight set to $\left\{w_{i j}\right\}_{(i, j) \in A}$ that all of them are greater or equal to one. Thus we can assume

$$
\sum_{i j} C_{i j} \cdot \bar{w}_{i j}=\bar{H} \text {. }
$$

Theorem 1: consider two optimization problems called DUAL_I and DUAL_II.

DUAL_I:

$$
\begin{gathered}
\max \sum_{k \in K} p_{t_{k}}^{k} \cdot D^{k} \\
p_{i}^{k}-p_{j}^{k} \leq w_{i j} \\
\sum_{(i, j) \in A} C_{i j} w_{i j}=1 \\
p_{s_{k}}^{k}=0 \\
w_{i j} \geq 0
\end{gathered}
$$

DUAL_II:

$$
\begin{gathered}
\max \sum_{k \in K} p_{t_{k}}^{\prime k} \cdot D^{k} \\
p_{i}^{\prime k}-p_{j}^{\prime k} \leq w_{i j}^{\prime} \\
\sum_{(i, j) \in A} C_{i j} w_{i j}^{\prime}=H \\
p_{s_{k}}^{\prime k}=0 \\
w_{i j}^{\prime} \geq 1
\end{gathered}
$$

If $\left\{\hat{w}_{i j}\right\}_{(i, j) \in A}$ is an optimal solution of DUAL_I and $\left\{\hat{w}_{i j}^{\prime}\right\}_{(i, j) \in A}$ is an optimal solution of DUAL_II, the sets $\left\{\hat{w}_{i j}\right\}_{(i, j) \in A}$ and $\left\{\hat{w}_{i j}^{\prime}\right\}_{(i, j) \in A}$ are equivalent weight sets with respect to $G(N, A)$.

Proof: Consider the optimization problem PRIMAL_II.

PRIMAL_II:

$$
\begin{gathered}
\max \sum_{k \in K} H \cdot p_{t_{k}}^{k} \cdot D^{k} \\
p_{i}^{k}-p_{j}^{k} \leq w_{i j} \\
\sum_{(i, j) \in A} C_{i j} w_{i j}=1 \\
p_{s_{k}}^{k}=0 \\
w_{i j} \geq 0
\end{gathered}
$$

It is clear that the $X_{i j}$ s which minimizes the objective function of the problem PRIMALL_II are the same as the ones which cause the problem PRIMALL_I to be optimized.

The dual of PRIMAL_II is the DUAL_TEMP.

DUAL_TEMP:

$$
\begin{gathered}
\max \sum_{k \in K} p_{t_{k}}^{\prime k} \cdot D^{k} \\
p_{i}^{\prime k}-p_{j}^{\prime k} \leq w_{i j}^{\prime} \\
\sum_{(i, j) \in A} C_{i j} w_{i j}^{\prime}=H \\
p_{s_{k}}^{\prime k}=0 \\
w_{i j}^{\prime} \geq 0
\end{gathered}
$$

From complementary slackness theorem

$$
\hat{X}_{i j}^{k} \cdot\left(\hat{p}_{i}^{{ }^{k}}-p_{j}^{\prime k}+w_{i j}^{\prime}\right)=0
$$

Since the optimal solutions of the PRIMAL_I and 
PRIMAL_II are the same, thus the weight sets $\left\{\hat{w}_{i j}\right\}_{(i, j) \in A}$ and $\left\{\hat{w}_{i j}^{\prime}\right\}_{(i, j) \in A}$ are equivalent weight sets. The weight set $\left\{\bar{w}_{i j}\right\}_{(i, j) \in A}$ is the feasible set of the problem DUAL_TEMP and hold (16). Therefore it is the optimal solution of this problem.

So we in this way, we were able to obtain optimal weights that do not include any link with weight 0 by limiting the constraint $w_{i j} \geq 0$ to $w_{i j}^{\prime} \geq 1$. This converts the problem DUAL_TEMP to DUAL_II. Figure 1 shows the flow chart of our method.

With ECMP routing a flow arriving at a node is split evenly over the links on the shortest paths from this node to the destination. It should be mentioned that arbitrary routing is not possible once ECMP in OSPF is used. so in OSPF environment we can never obtain the optimal routing but we can get close to it as much as possible.

Objective function that is used in [8] is min $M L U+r \sum_{k \in K} \sum_{(i, j) \in A} X_{i j}^{k}$. The second term in this objective function cause to minimizes $\sum X_{i j}^{k}$ in addition to $M L U$. In this case the weight ${ }^{i}$ siet $A^{A}$ resulted from the dual function is $\left\{w_{i j}+r\right\}$ (where $\left\{w_{i j}\right\}_{(i, j) \in A}$ are Lagrange multipliers that correspond to the non-equal constraint). The routing algorithm that we use in this paper is OSPF. This protocol splits the traffic equally among the available shortest paths, so we prefer traffic splitting as much as possible even if it passes through longer paths. As the constant $r$ in the second term prevents the flow to go through long paths we assume that $r=0$.

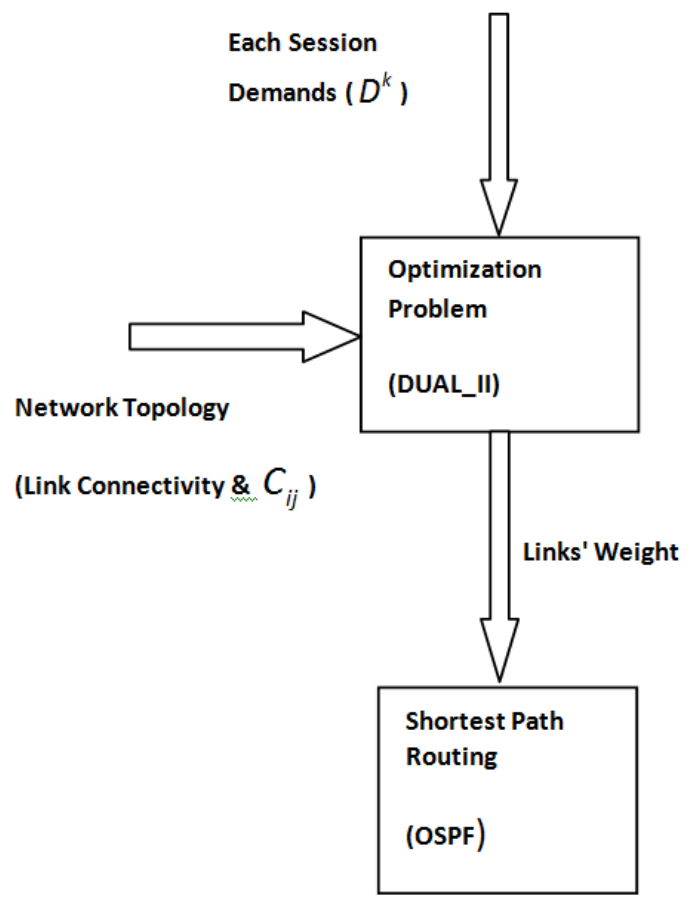

Figure 1. Maximum load balancing flow chart.

\section{Simulation Results}

In this section we simulate the OSPF protocol with its default link metrics and with the metrics that are calculated using the optimization problem.

A. Scenario I : In first scenario the simulation platform is shown in Figure 2.

All links in this network are DS3 with 44.7 Mbps rate. We suppose FIFO as a queuing policy. The session is a VOIP with GSM quality and the average bit rate is 40 Mbps. Node 1 is the source node and node 2 is the destination.

Table 1 show the solution of primal problem ( $X i j$ ) which indicate the paths that minimize maximum utilization. Solution of DUAL_I problem is shown in Table 2 and Table 3 show the solution of DUAL_II problem.

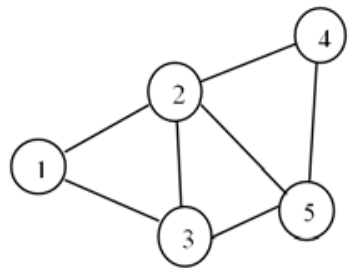

Figure 2. Simulation network topology.

Table 1. Optimum flows.

\begin{tabular}{cc}
\hline $\mathrm{X} 12$ & 20 \\
\hline $\mathrm{X} 13$ & 20 \\
$\mathrm{X} 23$ & 0 \\
$\mathrm{X} 24$ & 20 \\
$\mathrm{X} 25$ & 0 \\
$\mathrm{X} 35$ & 20 \\
$\mathrm{X} 54$ & 20 \\
\hline
\end{tabular}

Table 2. The Solution of DUAL_I.

\begin{tabular}{cc}
\hline w12 & 0.0056 \\
\hline w13 & 0.0017 \\
w23 & 0 \\
w24 & 0.0056 \\
w25 & 0 \\
w35 & 0.0077 \\
w54 & 0.0017 \\
\hline
\end{tabular}

Table 3. The Solution of DUAL_II.

\begin{tabular}{cc}
\hline W12 & 2.0177 \\
\hline W13 & 1.3567 \\
W23 & 1.0000 \\
W24 & 1.9823 \\
W25 & 1.0000 \\
W35 & 1.2843 \\
w54 & 0.0017
\end{tabular}


Table 1 show that the optimum paths are 1->2->4 and 1->3->5. These paths can obtain in a shortest path algorithm regarding to the weights that show in Table 2. Table 2 and Table 3 are the equivalent weights with respect to the graph that shows in Figure 2. So we use the suboptimal weigh set in Table $\mathbf{3}$ instead of default OSPF link metrics. Figure 3 show that in recent method packet drop decrease significantly.

In fowlloing scenarioes (senario 2-) the simulation platform is shown in Figure 4. We compare MLU, BWE (Bandwidth Efficiency), Number of Over Utilized Links, IP Traffic Dropped and IP Traffic Received for all scenarios

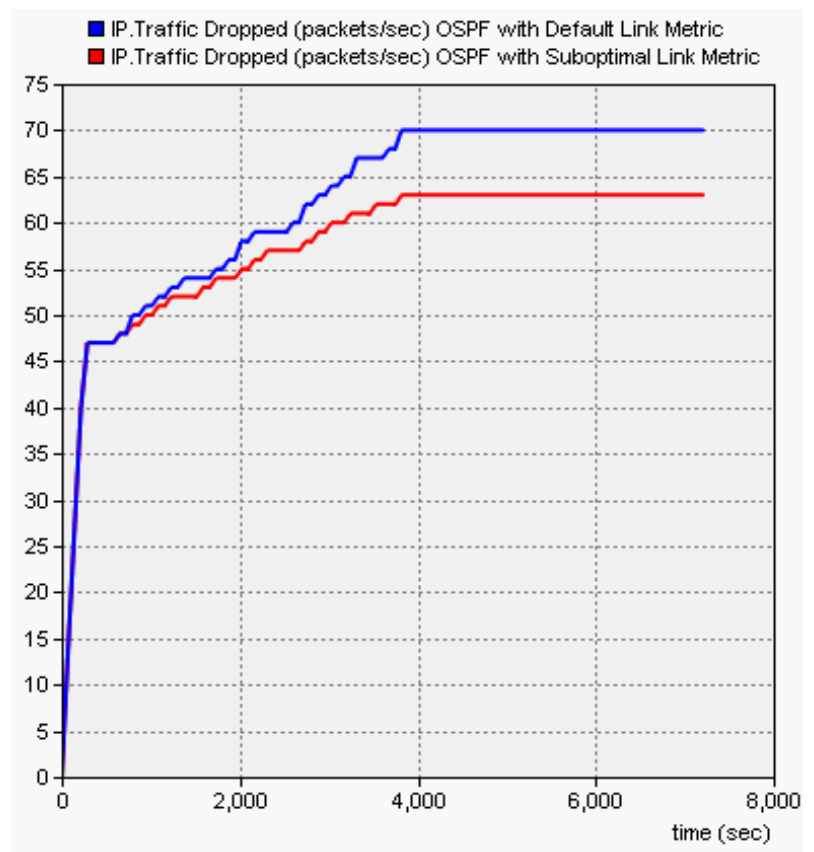

Figure.3. Dropping traffic in scenario1 for default protocol and new method.

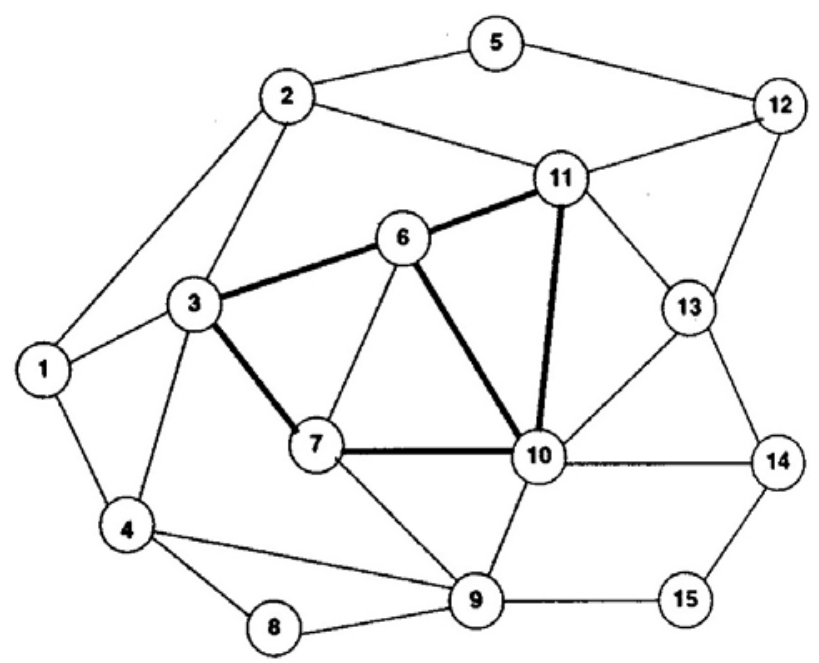

Figure 4. Simulation Network Topology.
In fowlloing scenarioes (senario 2-) the simulation platform is shown in Figure 4. We compare MLU, BWE (Bandwidth Efficiency), Number of Over Utilized Links, IP Traffic Dropped and IP Traffic Received for all scenarios

Scenario 2: In this scenario R1 is the traffic source and R13 is the traffic destination. Table $\mathbf{4}$ and Table $\mathbf{5}$ show the Suboptimal Link Weights that obtin by DUAL_II .

MLU in new method decreases from 91.3 percent to 36.3 percent and BWE increases from 7.6 percent to 10 percent as shown in Table 6.

Table 4. Solution of DUAL_II for Senario2.

\begin{tabular}{cccc}
\hline W1_2 & 56.84 & W3_6 & 1.000 \\
\hline W2_1 & 55.56 & W6_3 & 1.000 \\
W1_3 & 1.000 & W3_7 & 1.000 \\
W3_1 & 1.000 & W7_3 & 1.000 \\
W1_4 & 1.000 & W4_9 & 1.000 \\
W4_1 & 1.000 & W9_4 & 1.000 \\
W2_5 & 1.000 & W4_8 & 1.000 \\
W5_2 & 1.000 & W8_4 & 1.000 \\
W2_11 & 1.000 & W5_12 & 1.000 \\
W11_2 & 1.000 & W12_5 & 1.000 \\
W2_3 & 1.000 & W6_11 & 1.000 \\
W3_2 & 1.000 & W11_6 & 1.000 \\
W3_4 & 2.000 & W6_10 & 1.000 \\
W4_3 & 1.000 & W10_6 & 1.000 \\
\hline
\end{tabular}

Table.5. Solution of DUAL_II, cont for Senario2.

\begin{tabular}{cccc}
\hline W6_7 & 55.84 & W10_13 & 1.000 \\
\hline W7_6 & 1.000 & W13_10 & 1.000 \\
W7_10 & 1.000 & W10_14 & 1.000 \\
W10_7 & 1.000 & W14_10 & 1.000 \\
W7_9 & 1.283 & W11_12 & 1.000 \\
W9_7 & 1.000 & W12_11 & 1.000 \\
W8_9 & 1.000 & W11_13 & 1.000 \\
W9_8 & 1.000 & W13_11 & 1.000 \\
W9_10 & 1.000 & W12_13 & 1.000 \\
W10_9 & 1.000 & W13_12 & 1.000 \\
W9_15 & 2.000 & W13_14 & 1.000 \\
W15_9 & 1.000 & W14_13 & 1.000 \\
W10_11 & 1.000 & W14_15 & 1.000 \\
W11_10 & 1.000 & W15_14 & 1.000 \\
\hline
\end{tabular}

Table 6. MLU and BWE values for Senario2.

\begin{tabular}{ccc}
\hline & $\begin{array}{c}\text { Default } \\
\text { Algorithm }\end{array}$ & $\begin{array}{c}\text { Suboptimal } \\
\text { Algorithm }\end{array}$ \\
\hline MLU & 91.3 & 36.3 \\
BWE & 7.6 & 10 \\
Number of Over & 0 & 0 \\
Utilized Link & & \\
\hline
\end{tabular}


IP Traffic Dropped and IP traffic Received do not change in this scenario because there is no congestion

Scenario 3: in this scenario we have three source-destination pairs $\left(s_{1}, d_{1}\right),\left(s_{2}, d_{2}\right),\left(s_{3}, d_{3}\right)$ that are originated from R1 to R13, R5 to R9 and R4 to R2 respectively. MLU in the new method decreases from 137 percent to 91.3. The Number of over-utilized links also decreases from eight links to two links. BWE increases from 26.6 percent to 31.4 percent, Table 7. Figure 5 and Figure 6 show the comparison of IP Traffic Dropped and IP Traffic Received

\section{Conclusion}

In this paper we show that Optimization Theory can help Internet protocols work better. We use a duality theory to find a weight set that improve the routing protocols efficiencies. As a matter of fact routing is the most important aspect of Internet Traffic Engineering. So we focus on routing protocols and introduce a practical method that optimizes Link Metrics. Previous optimization methods suffer from practical issues but our method could be implemented with Routing Protocols that based on

Table 7. MLU and BWE values for Senario 3.

\begin{tabular}{ccc}
\hline & $\begin{array}{c}\text { Default } \\
\text { Algorithm }\end{array}$ & $\begin{array}{c}\text { Suboptimal } \\
\text { Algorithm }\end{array}$ \\
\hline MLU & 137 & 91.3 \\
BWE & 29.6 & 31.4 \\
$\begin{array}{c}\text { Number of Over } \\
\text { Utilized Link }\end{array}$ & 8 & 2 \\
\hline
\end{tabular}

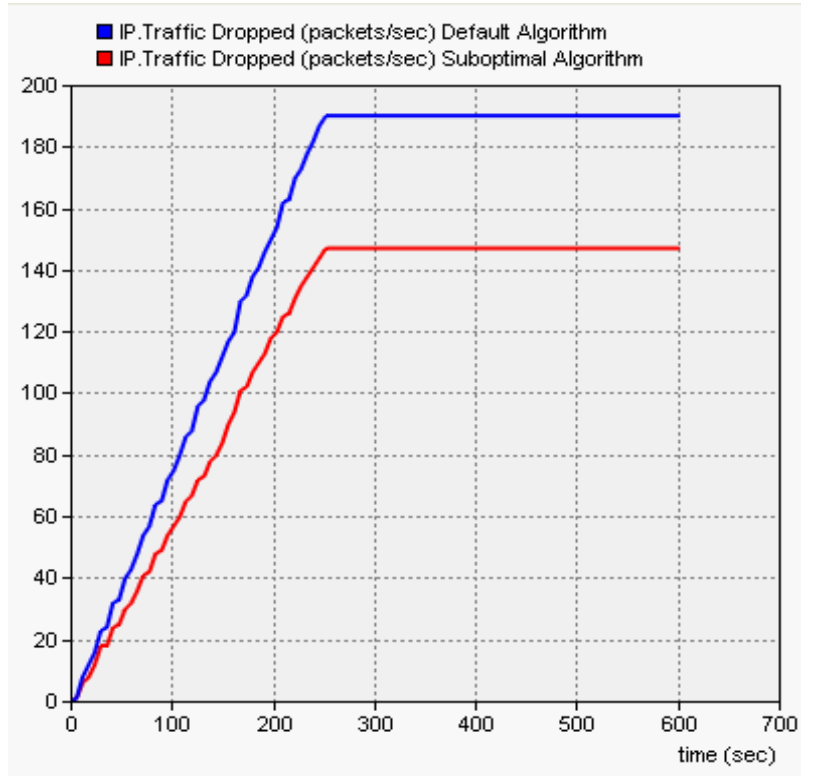

Figure 5. Ssenario2 IP traffic dropped.

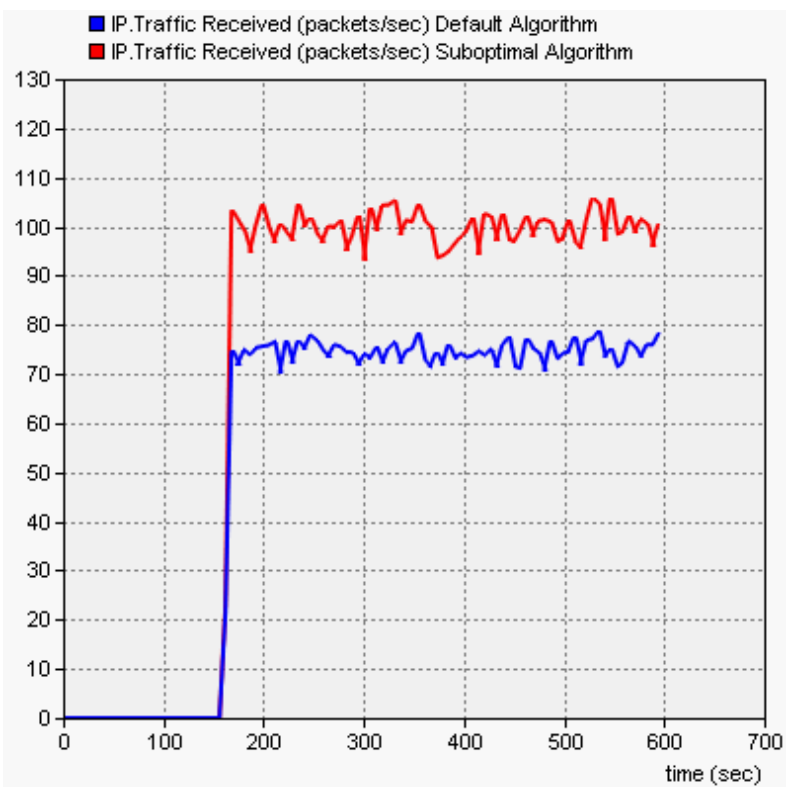

Figure 6. Ssenario2's IP traffic received.

shortest paths. Our simulation results show significant improvement on network efficiency.

\section{REFERENCES}

[1] Y. Lee et al., "Traffic Engineering in Next-Generation Optical Networks,” IEEE Commun. Surveys \& Tutorials, vol. 6, no. 3, 2004, pp. 16-33.

[2] D. Awduche et al, "Requirements on Traffic Engineering over. 2. MPLS,” RFC 2702, June 1999.

[3] D. Awduche et al., "MPLS and Traffic Engineering in IP Networks, IEEE Commun. Mag., vol. 37, no. 12, Dec. 1999, pp. 42-47.

[4] B. Fortz et al., "Internet Traffic Engineering by Optimising OSPF Weights,” Proc. IEEE INFOCOM, 2000, pp. 519-28.

[5] N. Hu et al., "Locating Internet Bottlenecks: Algorithms, Measure-ments and Implications," Proc. ACM SIGCOMM, 2004, pp. 41-54.

[6] A.Marija et al "Two Phase Load balance Routing using OSPF,” IEEE JOURNAL ON SELECTED AREAS IN COMMUNICATIONS, VOL. 28, NO. 1, JANUARY 2010

[7] E. Oki et al” Load-Balanced IP Routing Scheme Based on Shortest Paths in Hose Model" IEEE Transaction on comminications, Volume : 58, Page(s): 2088 - 2096, 2010

[8] Z. Wang, Y. Wang, and L. Zhang, ”Internet Traffic Engineering without Full Mesh Overlaying,” INFOCOM'2001

[9] S. Boyd and L. Vanderberghe. Convex Optimization. Cambridge Univ. Press, 2004

[10] D. P. Bertsekas. Network Optimization: Continuous and Discrete Models Athena Scientific, 1998 